\title{
Gauge dependence of tadpole and mass renormalization for a seesaw extended $2 \mathrm{HDM}$
}

\author{
Vytautas Dūdènas ${ }^{*}$ and Thomas Gajdosik \\ Institute of Theoretical Physics and Astronomy, Faculty of Physics, Vilnius University, \\ Sauletekio avenue 3, 10257 Vilnius, Lithuania
}

(Received 22 June 2018; published 24 August 2018)

\begin{abstract}
We study the gauge dependence of the neutrino mass renormalization in a two Higgs doublet model, that is extended with one singlet seesaw neutrino. This model gives only one light neutrino a mass at tree level, while the second light mass is generated at loop level via the interaction with the second Higgs doublet. At one loop level, one neutrino stays massless. We use multiplicative renormalization constants to define counterterms. The renormalized mass parameters are defined as the complex poles of the propagators, using the complex mass scheme for mass renormalization. With this setup, we analytically get the expressions for the neutrino mass counterterms and isolate the gauge dependent part. We show, how relating this gauge dependent part with the tadpole renormalization leads to gauge independent counterterm definitions, hence gauge independent bare masses for neutrinos.
\end{abstract}

DOI: 10.1103/PhysRevD.98.035034

\section{INTRODUCTION}

Neutrino oscillations are known for more than 30 years [1]. They prove that neutrinos are not massless. However, how exactly neutrinos get their masses in the framework of quantum field theory is still unclear. Seesaw mechanisms $[2,3]$ are by far the most popular attempts to extend the standard model with massive neutrinos. The type I seesaw mechanism [2] is the earliest and simplest such extension, which includes neutrino mass terms induced by the Higgs boson of the standard model (SM). In case there are more Higgs bosons than the single SM Higgs, the type I seesaw extension can be generalized as in [4]. This allows for a wider range of configurations in the seesaw and Yukawa sectors to generate the masses for neutrinos that are in agreement with the experimental values. Also, there are numerous theoretical motivations [5-8] suggesting a larger scalar sector. We restrict ourselves to a general $C P$ conserving two Higgs doublet model (2HDM) [9], which can be viewed as a general class of more specific models that include two scalar doublets under the gauge group $S U(2)_{\text {weak }}$.

The 2HDM paired with the seesaw mechanism gives a new way of generating masses for neutrinos that is absent in the usual SM seesaw extensions. That is, the mass terms that are absent at tree level arise at loop level due to the

\footnotetext{
*vytautasdudenas@inbox.lt
}

Published by the American Physical Society under the terms of the Creative Commons Attribution 4.0 International license. Further distribution of this work must maintain attribution to the author(s) and the published article's title, journal citation, and DOI. Funded by SCOAP ${ }^{3}$. interactions with the second Higgs doublet. This radiative mass generation makes it possible to account for both experimentally measured mass differences at one loop level having only one sterile neutrino in the seesaw mechanism. This set up, with the 2HDM and one sterile neutrino at one loop was first proposed in [4] and we call it the GrimusNeufeld model (GN model).

We look at the gauge parameter dependence of the neutrino mass renormalization in this GN model with a $C P$ symmetric $2 \mathrm{HDM}$ potential. It is proven in general [10], that the position of the complex pole of the propagator is independent of the gauge. Hence one can extend the onshell (OS) scheme to the complex domain to define gauge invariant masses as is done in the complex mass scheme (CMS) $[11,12]$. However, this does not mean that the mass counterterms are necessarily gauge parameter independent. In fact, at one loop there is the same gauge dependence of the mass counterterms in the CMS as in the OS scheme. This is because the one loop expressions for the OS are the same as in the CMS except for the required reality of loop functions in the OS scheme. As long as the mass is evaluated at the exact pole (as in the CMS), this gauge dependence of the counterterm does not bother the definition of mass since the exact pole is gauge independent anyway. Defining a gauge independent counterterm, however, is important in other schemes such as (modified) minimal subtraction, where the gauge dependence might occur in the running of parameters [13,14]. Some explicit examples of the gauge dependence in the $\overline{\mathrm{MS}}$ scheme are given in [15-17]. Hence it is worth to look at the possibilities to define gauge independent mass counterterms in the CMS or the OS, as well. 
In the GN model, we analytically check that the gauge dependent terms for the fermion two point function vanish if the tadpole diagrams are attached to the propagator as discussed in [18]. This way of dealing with gauge dependent parts originates from the pinch technique [19]. Hence applying this technique to define numerically gauge invariant counterterms seems rather straightforward. However, to analytically isolate these tadpole diagrams from the counterterms requires some effort. We present how we achieve this isolation of the gauge dependent terms for the neutrino mass counterterms in the GN model. We try to be as transparent as possible in showing our steps so that the reader can easily reproduce our results. All our renormalization constants arise from multiplicative renormalization and we use Weyl spinors for our expressions rather than Dirac spinors.

In Sec. II we present the main definitions and discuss the implications of using the complex mass scheme over the on-shell scheme. In Sec. III we introduce the scalar sector and present the tadpole renormalization conditions in the 2HDM. In Sec. IV we introduce the Yukawa sector of the GN model and show the expressions of mass counterterms for neutrinos. The relationship between tadpole conditions of Sec. III and mass counterterms is also explained in Sec. IV. In Sec. V we show how we set up the calculations using SARAH [20](version 4.12.0 [21]), FEYNARTS [22] (version 3.9) and FoRMCALC [23] (version 9.4) and present the analytical results. Section V is accompanied by the Appendix B in which we present some intermediate steps of the derivations. We conclude the results in Sec. VI by discussing the cancellation of the gauge dependence of neutrino propagators in the GN model.

\section{DEFINITIONS AND THE COMPLEX MASS SCHEME}

We use the same definitions as in [24], where we presented the adaptation of the complex mass scheme [12] for Majorana fermions in Weyl spinor formalism. The renormalized Green functions are

$$
\begin{aligned}
\frac{1}{i}\left\langle\phi_{1} \ldots \phi_{n}\right\rangle_{1 P I}^{[\text {loop }]} & =\left.\frac{\delta^{n} \hat{\Gamma}^{[\text {loop }]}}{\delta \phi_{1} . . \delta \phi_{n}}\right|_{\phi_{i}=0} \equiv \hat{\Gamma}_{\phi_{1} \ldots \phi_{n}}^{[\text {loop }]} \\
& \equiv \Gamma_{\phi_{1} \ldots \phi_{n}}^{[\text {loop }]}+\delta \Gamma_{\phi_{1} \ldots \phi_{n}}^{[\text {loop }]},
\end{aligned}
$$

where $\delta \Gamma^{[\text {loop }]}$ stands for the counterterm part of the renormalized effective action. The superscript denotes the loop order of the function in consideration. The tadpole function is defined as the special case of Eq. (1):

$$
T_{\phi}^{[\mathrm{loop}]} \equiv \Gamma_{\phi}^{[\mathrm{loop}]} .
$$

The definitions for using Weyl spinors as the basis of Feynman diagram calculations can be found in [25]. The scalar parts of Green's functions of a left handed Weyl spinor $\nu_{i}$ and its Hermitian conjugate $\nu_{i}^{\dagger}$ can be separated by the Lorentz index structure:

$$
\begin{array}{ll}
\hat{\Gamma}_{\nu_{i} \nu_{i}}=m_{i} \hat{\Sigma}_{\nu_{i} \nu_{i}}, & \hat{\Gamma}_{\nu_{i}^{\dagger} \nu_{i}^{\dagger}}=m_{i} \hat{\Sigma}_{\nu_{i}^{\dagger} \nu_{i}^{\dagger}}, \\
\hat{\Gamma}_{\nu_{i} \nu_{j}^{\dagger}}=p \sigma \hat{\Sigma}_{\nu_{i} \nu_{j}^{\dagger}}, & \hat{\Gamma}_{\nu_{i}^{\dagger} \nu_{j}}=p \bar{\sigma} \hat{\Sigma}_{\nu_{i}^{\dagger} \nu_{j}} .
\end{array}
$$

The definitions of Eq. (3) work well for the on-shell scheme, but have to be slightly modified for the complex mass scheme.

We work in renormalized perturbation theory, where the renormalized parameters $p$ and the renormalized fields $\phi_{j}$ are related to bare parameters and bare fields by multiplicative renormalization constants:

$$
p_{0}=p\left(1+\delta_{p}\right), \quad \phi_{0 i}=\sum_{j}\left(1_{i j}+\delta_{i j}\right) \phi_{j} .
$$

We use the subscript 0 to denote the bare quantities, $1_{i j}$ stands for the Kronecker delta, $\delta_{p}$ and $\delta_{i j}$ are one loop order renormalization constants. These redefinitions of parameters and fields give rise to the counterterms $\delta \Gamma_{\phi_{1} \ldots \phi_{n}}^{[\text {loop }}$ in Eq. (1).

We use the general $R_{\xi}$ gauge for calculations. As we will look at the gauge parameter dependencies, we will frequently look at only the gauge parameter dependent part of the expressions. To denote the gauge dependent term, we will add the gauge parameter $\xi$ in the subscript at the end of the renormalization constants, self-energies, and tadpole functions; e.g.:

$\delta_{p} \equiv \delta_{p \xi}+$ gauge independent terms, $\quad \delta_{p \xi}=\delta_{p \xi_{W}}+\delta_{p \xi_{Z}}$.

We use the complex mass scheme [12] (CMS) to renormalize masses and fields. The CMS for mixed fermions is presented in [26-28] and the adaptation to Weyl spinor formulation is presented in [24]. Here we mention the main differences that need to be considered when generalizing the OS framework to the CMS. Considering a Majorana mass term for the Weyl fermion $\nu$ :

$$
\mathcal{L}_{m_{0}}=-\frac{1}{2} m_{0} \nu_{0} \nu_{0}-\frac{1}{2} m_{0}^{\dagger} \nu_{0}^{\dagger} \nu_{0}^{\dagger},
$$

we can absorb the phase of the mass parameter into the field, so that $m_{0} \in \mathbb{R}$ :

$$
\mathcal{L}_{m_{0}}=-\frac{1}{2} m_{0}\left(\nu_{0} \nu_{0}+\nu_{0}^{\dagger} \nu_{0}^{\dagger}\right)
$$

Renormalizing the mass parameter leads to

$$
\mathcal{L}_{m_{0}}=-\frac{1}{2} m\left(\nu_{0} \nu_{0}+\nu_{0}^{\dagger} \nu_{0}^{\dagger}\right)+\text { c.t. },
$$

where $m \in \mathbb{C}$ and c.t. stands for the counterterms. Hence the CMS introduces an apparent nonhermiticity in the 
renormalized tree level Lagrangian (the full Lagrangian including all the counterterms is Hermitian). Also, the condition for the residue at the complex pole leads to an additional phase difference in the fields [26-28]. That means that the field renormalization constants are not Hermitian conjugate to each other either [24]:

$$
\nu_{0}^{\dagger}=(1+\bar{\delta}) \bar{\nu}, \quad \nu_{0}=(1+\delta) \nu \Rightarrow \bar{\nu} \neq \nu^{\dagger}, \quad \delta^{\dagger} \neq \bar{\delta},
$$

where we use overbars as parts of the names of the renormalization constants and the fields. Hence the renormalized mass Lagrangian in the CMS is:

$$
\mathcal{L}_{m}=-\frac{1}{2} m(\nu \nu+\bar{\nu} \bar{\nu})
$$

Comparing with the bare Lagrangian, we see that we could write Eq. (6) or Eq. (7) as:

$$
\mathcal{L}_{m_{0}}=-\frac{1}{2} m_{0} \nu_{0} \nu_{0}+\text { H.c. }
$$

We cannot write Eq. (10) in the same way, since it is not hermitian. However, we can try to define a new symbol H.c. ${ }^{*}$ to have the possibility to write:

$$
\mathcal{L}_{m}=-\frac{1}{2} m(\nu \nu+\bar{\nu} \bar{\nu})=-\frac{1}{2} m \nu \nu+\text { H.c.* }
$$

In this equation the symbol H.c.* makes the replacement for the field $\nu \rightarrow \bar{\nu}$ and leaves $m \rightarrow m$. The mass parameter is unchanged in the H.c. ${ }^{*}$ since we found the basis, in which the bare parameter is real by absorbing the phase into $\nu_{0}$ in Eq. (7). Hence the algebraic structure of Eq. (7) is kept in the renormalized version shown in Eq. (10). A similar thing happens in the $C P$ conserving Higgs sector: the $C P$ symmetry constrains the form of the Lagrangian, which has to be kept during the renormalization condition. Also, in the scalar and the vector case, if we have $\phi_{0} \in \mathbb{R}$, then $\phi=\bar{\phi}$. The easiest way to generalize the H.c. ${ }^{*}$ symbol is to say that we choose the basis in which the bare parameters that can be real are made real; then we can summarize:

$$
\text { H.c.* }: \begin{cases}p \rightarrow p, \phi \rightarrow \bar{\phi} ; & p_{0} \in \mathbb{R} \\ p \rightarrow p^{\dagger}, \phi \rightarrow \bar{\phi} ; & p_{0} \notin \mathbb{R} .\end{cases}
$$

Normally, if a bare parameter is related to the bare mass term, that parameter can be made real by absorbing the phase into the field. Hence the second line of Eq. (13) assumes that there is no effect of the mass renormalization to the parameter $p$ if $p_{0}$ cannot be related to the mass term. While this assumption is correct at one loop level, the definition Eq. (13) at higher loops should be treated with caution. Without going into too much technical details, one can think of H.c. ${ }^{*}$ as a shorthand notation for the renormalized H.c. terms of the bare Lagrangian.
Now we can come back to the definitions of Eq. (3). As the CMS renormalized field is $\bar{\nu}$ and not $\nu^{\dagger}$, as can be seen from Eq. (9), we write [24]:

$$
\begin{array}{ll}
\hat{\Gamma}_{\nu_{i} \nu_{i}}=m_{i} \hat{\Sigma}_{\nu_{i} \nu_{i}}, & \hat{\Gamma}_{\bar{\nu}_{i} \bar{\nu}_{i}}=m_{i} \hat{\Sigma}_{\bar{\nu}_{i} \bar{\nu}_{i}}, \\
\hat{\Gamma}_{\nu_{i} \bar{\nu}_{j}}=p \sigma \hat{\Sigma}_{\nu_{i} \bar{\nu}_{j}}, & \hat{\Gamma}_{\bar{\nu}_{i} \nu_{j}}=p \bar{\sigma} \hat{\Sigma}_{\bar{\nu}_{i} \nu_{j}} .
\end{array}
$$

The difference between Eqs. (3) and (14) is rather formal: i.e., one does not really see the difference when calculating the Feynman diagrams. However, for using the CMS for field and mass renormalization, one should keep this difference in mind for the conceptual consistency.

After we have the consistent set up for renormalizing the fermions in the CMS, we continue to look at the gauge parameter dependencies of the renormalization constants in this scheme. The multiplicative renormalization constants Eq. (4) can be used for any renormalization condition. The algebra of the CMS is basically the same as in the OS, as the CMS is just the analytical continuation of the OS to the complex domain. In this paper, we study the algebraic relations that allow to isolate the gauge parameter term in the mass counterterm. As this procedure is purely algebraic, the expressions concerning the isolation of the gauge dependent part are the same as in the OS scheme apart from the reality requirement. We, however, do these manipulations with the CMS in mind, as the generalizations despite being rather straightforward are still needed for a full consistency. We now turn to the explicit expressions for the GN model.

\section{SCALAR SECTOR AND TADPOLE CONDITIONS}

The general 2HDM is an extension of the SM with a second Higgs doublet having the same charges as the SM Higgs doublet. The most general potential can be written as $[9,29]$ :

$$
\begin{aligned}
\mathcal{V}_{\text {Higgs }} & =m_{011}^{2} H_{01}^{\dagger} H_{01}+m_{022}^{2} H_{02}^{\dagger} H_{02}-\left(m_{012}^{2} H_{01}^{\dagger} H_{02}+\text { H.c. }\right) \\
& +\frac{1}{2} \lambda_{01}\left(H_{01}^{\dagger} H_{01}\right)^{2}+\frac{1}{2} \lambda_{02}\left(H_{02}^{\dagger} H_{02}\right)^{2} \\
& +\lambda_{03}\left(H_{01}^{\dagger} H_{01}\right)\left(H_{02}^{\dagger} H_{02}\right)+\lambda_{04}\left(H_{02}^{\dagger} H_{01}\right)\left(H_{01}^{\dagger} H_{02}\right) \\
& +\left[\frac{1}{2} \lambda_{05}\left(H_{02}^{\dagger} H_{01}\right)\left(H_{02}^{\dagger} H_{01}\right)+\lambda_{06}\left(H_{01}^{\dagger} H_{01}\right)\left(H_{01}^{\dagger} H_{02}\right)\right. \\
& \left.+\lambda_{07}\left(H_{02}^{\dagger} H_{02}\right)\left(H_{02}^{\dagger} H_{01}\right)+\text { H.c. }\right],
\end{aligned}
$$

where $H_{01}$ and $H_{02}$ are the two Higgs doublets. In a general basis, they both develop VEVs: $v_{01}$ and $v_{02}$, respectively. The VEV value that is responsible for the electroweak symmetry breaking is $v_{0}^{2}=v_{01}^{2}+v_{02}^{2}$. We choose to work in the Higgs basis, where we can parametrize the Higgs doublets as: 


$$
\begin{aligned}
& H_{01}=\left(\begin{array}{c}
\chi_{0 W}^{+} \\
\frac{1}{\sqrt{2}}\left(v_{0}+h_{0}+i \chi_{0 Z}\right)
\end{array}\right), \\
& H_{02}=\left(\begin{array}{c}
H_{0}^{+} \\
\frac{1}{\sqrt{2}}\left(H_{0}+i A_{0}\right)
\end{array}\right) .
\end{aligned}
$$

In this basis, $H_{02}$ is chosen to have 0 vacuum expectation value (VEV), $v_{0}$ is the VEV of $H_{01}, \chi_{0 Z}$, and $\chi_{0 W}$ stand for Goldstone bosons, $h_{0}, H_{0}$, and $A_{0}$ are neutral scalars and $H_{0}^{+}$is a charged scalar. Note that when we choose the Higgs basis by Eq. (16) and insert into the Eq. (15), the parameters in Eq. (15) are the Higgs basis parameters and not the ones of the general basis. The transformation of parameters between the Higgs and the general basis can be found in $[9,30]$. We consider the $C P$ conserving case, where all the bare parameters are real,

$$
m_{0 i j}^{2}, \lambda_{0 k} \in \mathbb{R} ; \quad i, j=1,2, \quad k=1, \ldots, 7,
$$

by an imposed $C P$ symmetry on the bare Lagrangian.

After introducing the renormalization constants, Eq. (4), we write the zeroth order renormalized effective action (or the renormalized Lagrangian, ignoring the kinetic terms) of the Higgs sector as:

$$
\begin{aligned}
\Gamma_{\text {Higgs }}^{[0]}= & -m_{11}^{2} \bar{H}_{1} H_{1}-m_{22}^{2} \bar{H}_{2} H_{2}+\left\{m_{12}^{2} \bar{H}_{1} H_{2}+\text { H.c. }{ }^{*}\right\} \\
& -\frac{1}{2} \lambda_{1}\left(\bar{H}_{1} H_{1}\right)^{2}-\frac{1}{2} \lambda_{2}\left(\bar{H}_{2} H_{2}\right)^{2}-\lambda_{3}\left(\bar{H}_{1} H_{1}\right)\left(\bar{H}_{2} H_{2}\right) \\
& -\lambda_{4}\left(\bar{H}_{2} H_{1}\right)\left(\bar{H}_{1} H_{2}\right) \\
& -\left[\frac{1}{2} \lambda_{5}\left(\bar{H}_{2} H_{1}\right)\left(\bar{H}_{2} H_{1}\right)+\lambda_{6}\left(\bar{H}_{1} H_{1}\right)\left(\bar{H}_{1} H_{2}\right)\right. \\
& \left.+\lambda_{7}\left(\bar{H}_{2} H_{2}\right)\left(\bar{H}_{2} H_{1}\right)+\text { H.c. } .\right],
\end{aligned}
$$

where we used the definitions of Eq. (13). As the bare fields $h_{0}, H_{0}, A_{0}$ are real, the renormalized fields are written as:

$$
\begin{aligned}
& H_{1}=\left(\begin{array}{c}
\chi_{W}^{+} \\
\frac{1}{\sqrt{2}}\left(v+h+i \chi_{Z}\right)
\end{array}\right), \quad H_{2}=\left(\begin{array}{c}
H^{+} \\
\frac{1}{\sqrt{2}}(H+i A)
\end{array}\right), \\
& \bar{H}_{i}=H_{i}^{T}\left(\chi^{+} \rightarrow \chi^{-}, H^{+} \rightarrow H^{-}, i \rightarrow-i\right) .
\end{aligned}
$$

$\chi_{W}^{+}$and $\chi_{W}^{-}$are related to $\chi_{0 W}^{+}$as described by Eq. (9). The same holds for $\mathrm{H}^{+}$and $\mathrm{H}^{-}$. The neutral fields appear in the barred doublets in the same way as in the unbarred doublets.

To get the minimum of the potential, Eq. (18), we need to solve three tadpole equations for the three neutral scalars. It is important to note that we will express the tadpole equations in the Higgs basis and not in the mass eigenstate basis as the expressions are simpler. The mass eigenstate basis for $h$ and $H$ and the Higgs basis is related by an orthogonal transformation parametrized by [9]:

$$
\begin{aligned}
& O^{\phi}=\left(\begin{array}{cc}
c_{\alpha} & s_{\alpha} \\
-s_{\alpha} & c_{\alpha}
\end{array}\right), \quad \phi_{i}^{\text {mass }}=O_{i j}^{\phi} \phi_{j}^{\text {Higgs }}, \\
& \phi_{i}^{\text {Higgs }}=(h, H)_{i},
\end{aligned}
$$

where $s_{\alpha}$ and $c_{\alpha}$ are sine and cosine functions of a mixing angle $\alpha$, respectively. In general, we would have $3 \times 3$ mixing matrix, but the imposed $C P$ symmetry on the potential does not allow $A$ to mix with $h$ and $H$ at tree level. Then the tadpole functions in different bases are related by:

$T_{h}=c_{\alpha} T_{h_{(m)}}-s_{\alpha} T_{H_{(m)}}, \quad T_{H}=c_{\alpha} T_{H_{(m)}}+s_{\alpha} T_{h_{(m)}}$,

$T_{A}=T_{A_{(m)}}$,

where we added the $m$ in the subscript to indicate that the fields are in the mass eigenstates. At tree level, the tadpole functions are

$$
\begin{aligned}
& \hat{T}_{h}^{[0]}=\frac{\delta \Gamma_{\text {Higgs }}^{[0]}}{\delta h}=-v\left(m_{11}^{2}+\frac{1}{2} \lambda_{1} v^{2}\right), \\
& \hat{T}_{H}^{[0]}=\frac{\delta \Gamma_{\text {Higgs }}^{[0]}}{\delta H}=v\left(m_{12}^{2}-\frac{1}{2} v^{2} \lambda_{6}\right), \\
& \hat{T}_{A}^{[0]}=\frac{\delta \Gamma_{\text {Higgs }}^{[0]}}{\delta A}=0 .
\end{aligned}
$$

We see that the third tadpole function is already zero in the $C P$ conserving case. We require the tadpole conditions to hold for all loop levels:

$$
\hat{T}_{h}^{[i]}=\hat{T}_{H}^{[i]}=\hat{T}_{A}^{[i]}=0 .
$$

The tree level tadpole conditions $\hat{T}_{h}^{[0]}=\hat{T}_{H}^{[0]}=\hat{T}_{A_{0}}^{[0]}=0$ give:

$$
m_{11}^{2}=-\frac{1}{2} \lambda_{1} v^{2} \quad \text { and } \quad m_{12}^{2}=\frac{1}{2} \lambda_{6} v^{2} .
$$

Now we require the tadpole conditions Eq. (23) for tree and one loop level together:

$$
\hat{T}^{[0]}=0,\left.\quad\left(T^{[1]}+\delta \hat{T}^{[1]}\right)\right|_{\hat{T}^{[0]}=0}=0,
$$

where we indicate in the second equation that we use the relations from the first condition at the loop order after algebraically deriving counterterms from the multiplicative constants shown in Eq. (4). The one loop tadpole counterterms evaluated at $\hat{T}^{[0]}=0$ for the $C P$ conserving case then are 


$$
\begin{aligned}
\delta \hat{T}_{h}^{[1]} & =\frac{1}{2} \lambda_{1} v^{3}\left(2 \delta_{m 11}-\delta_{\lambda 1}-2 \delta_{v}\right), \\
\delta \hat{T}_{H}^{[1]} & =\frac{1}{2} \lambda_{6} v^{3}\left(2 \delta_{m 12}-\delta_{\lambda 6}-2 \delta_{v}\right), \\
\delta \hat{T}_{A_{0}}^{[1]} & =0 .
\end{aligned}
$$

As $v$ is defined dynamically by Eq. (24), it is not an independent parameter of the theory. This means that one of the counterterms $\delta_{m 11}, \delta_{\lambda 1}, \delta_{v}$ is redundant. This is because we did not yet choose which parameter is used over which from the tree level minimum condition Eq. (24). One of the choices is treating $\lambda_{1}$ and $v$ as the independent ones so that the shift of $m_{11}$ is given by:

$$
\delta_{m 11}=\frac{1}{2} \delta_{\lambda 1} .
$$

Then the shift of the VEV yields the one loop tadpole counterterms, evaluated at $\hat{T}^{[0]}=0$ :

$$
\begin{gathered}
\delta \hat{T}_{h}^{[1]}=-\lambda_{1} v^{3} \delta_{v}, \\
\delta \hat{T}_{H}^{[1]}=\frac{1}{2} \lambda_{6} v^{3}\left(2 \delta_{m 12}-\delta_{\lambda 6}-2 \delta_{v}\right) .
\end{gathered}
$$

The one loop tadpole conditions Eq. (25) give:

$$
\begin{gathered}
\delta_{v}=\frac{1}{\lambda_{1} v^{3}} T_{h}^{[1]}, \\
\left(\delta_{m 12}-\frac{1}{2} \delta_{\lambda 6}\right)=\frac{1}{v^{3}}\left(\frac{1}{\lambda_{1}} T_{h}^{[1]}-\frac{1}{\lambda_{6}} T_{H}^{[1]}\right) .
\end{gathered}
$$

The $v$ now stands for a loop renormalized VEV or the "proper VEV" as in [31]. So far, the construction is similar to the $\beta_{t}$ scheme of [32], "scheme 3" in [13] or [31] of the $\mathrm{SM}$, but without the proper relation of the VEV to the mass terms, it is not yet complete. To complete it as in [13,31,32], one identifies the bare mass parameters arising from the proper VEV, rather than $v_{0}$, as also noted in [13,31-35]. The idea is to avoid the inclusion of the gauge dependence coming from $\delta_{v}$ into the definition of the mass counterterm $\delta_{m}$ as will be shown in the next sections.

\section{YUKAWA SECTOR}

The GN model adds a single sterile neutrino $N_{0}$ to the general 2HDM. This sterile neutrino is a gauge singlet under all gauge groups of the SM and has a Majorana mass term $M_{0}$. To write the Yukawa couplings, we start in the flavor basis, in which the Yukawa coupling of the charged fermions to the first Higgs doublet in the Higgs basis is diagonal. Then the general Yukawa couplings for neutrinos can be seen as two three-vectors $Y^{1}$ and $Y^{2}$. The neutrino Yukawa Lagrangian together with the Majorana mass term then is written as:
$\mathcal{L}_{\text {Yuk }}=-Y_{i}^{1} n_{0 i} N_{0} H_{01}-Y_{i}^{2} n_{0 i} N_{0} H_{02}-\frac{1}{2} M_{0} N_{0} N_{0}+$ H.c.

where $n_{0 i}$ are neutrinos in the flavor basis with $i=e, \mu, \tau$. The Yukawa couplings $Y_{i}^{1}$ and $Y_{i}^{2}$ give in general 6 complex parameters and $M_{0}$ gives 1 complex parameter. We absorb four phases into the $n_{0 i}$ and $N_{0}$ to get $Y_{i}^{1}, M_{0} \in \mathbb{R}$. By a singular value decomposition, we can parametrize the Yukawa couplings with only four real parameters:

$$
d_{0}, y_{0} \in \mathbb{R}, \quad d_{0}^{\prime} \in \mathbb{C},
$$

absorbing the other degrees of freedom into the Unitary mixing matrix. To make the parametrization easy, we decompose it into subsequent orthogonal rotations $O$ and phase shifts $U$, so that $O^{23}$ produces zero in the second position of $Y^{1}\left(O_{2 j}^{23} Y_{j}^{1}=0\right), O^{13}$ in the first $\left(O_{1 k}^{13} O_{k j}^{23} Y_{j}^{1}=\right.$ $0)$. $U^{\sigma}$ adjusts the phase of the first element of $Y^{2}$ to match it with the phase of the second element $\left(\arg \left(U_{1 l}^{\alpha} O_{l k}^{13} O_{k j}^{23} Y_{j}^{2}\right)=\arg \left(U_{2 l}^{\alpha} O_{l k}^{13} O_{k j}^{23} Y_{j}^{2}\right), \quad O^{12}\right.$ makes the first element of $Y^{2}$ zero $\left(O_{1 m}^{12} U_{m l}^{\alpha} O_{l k}^{13} O_{k j}^{23} Y_{j}^{2}=0\right)$ and $U^{\rho}$ adjust the phase so that the second element of $Y^{2}$ is real $\left(U_{2 n}^{\beta} O_{n m}^{12} U_{m l}^{\alpha} O_{l k}^{13} O_{k j}^{23} Y_{j}^{2} \in \mathbb{R}\right)$. Writing $V=U^{\beta} O^{12} U^{\alpha} O^{13} O^{23}$, the basis choice is summarized as:

$V_{1 j} Y_{j}^{1}=0, \quad V_{2 j} Y_{j}^{1}=0, \quad V_{3 j} Y_{j}^{1}=y_{0}$,

$V_{1 j} Y_{j}^{2}=0, \quad V_{2 j} Y_{j}^{2}=d_{0}, \quad V_{2 j} Y_{j}^{2}=d_{0}^{\prime}$,

$d_{0}, y_{0} \in \mathbb{R}, \quad d_{0}^{\prime} \in \mathbb{C}$.

Note that we are still free to adjust the phase of the first row of $V$. To combine these rotations with the seesaw transformation, we combine all neutrinos to a single vector, consisting of four flavor basis neutrinos:

$$
\nu_{0 i}^{F}=\left(n_{0 e}, n_{0 \mu}, n_{0 \tau}, N_{0}\right)_{i} .
$$

To account for the fourth component of this vector, the $3 \times 3$ matrix $V$ is trivially extended to a $4 \times 4$ matrix by adding an identity element on the diagonal. As we work in the Higgs basis, only the first Higgs doublet gets the VEV. With the parametrization Eq. (34), the seesaw transformation acts on the third and fourth component yielding the whole $4 \times 4$ mixing matrix:

$$
U=U^{34} V=U^{34} U^{\beta} O^{12} U^{\alpha} O^{13} O^{23}
$$

and the relation between the mass eigenstate and the flavor basis becomes:

$$
\nu_{0 i}^{\operatorname{mass}}=U_{i j}^{*} \nu_{0 j}^{F} .
$$


All the parametrization of neutrino mixing matrix is summarized in Appendix A.

In order to see the differences in the mass terms between the tadpole schemes, we first do the usual construction like in, e.g., [12], and then modify it according to the discussion at the end of Sec. III. After the electroweak symmetry breaking, the seesaw mechanism yields two bare mass eigenvalues $m_{03}$ and $m_{04}$ that have the relations:

$$
M_{0}=m_{04}-m_{03} \quad \text { and } \quad y_{0}^{2} v_{0}^{2}=2 m_{03} m_{04} .
$$

The seesaw parameters are expressed in terms of masses:

$$
s_{034}^{2}=\frac{m_{03}}{m_{04}+m_{03}} \quad \text { and } \quad c_{034}^{2}=\frac{m_{04}}{m_{04}+m_{03}} .
$$

Note that as long as we stay at tree level, $v_{0}=v$. In this basis we have four neutrino states $\nu_{0 i}$, where $\nu_{01}$ and $\nu_{02}$ have zero mass, but $\nu_{02}$ is distinguished from $\nu_{01}$ by its interaction with the second Higgs doublet, i.e., $\nu_{01}$ does not couple to any of the Higgses. By applying the rotation Eq. (37) in Eq. (32), using the parametrizations of Eq. (34), (38), and (39) and inserting the explicit Higgs basis Eq. (16), we write the Yukawa Lagrangian part that includes only neutral scalar fields together with the Majorana mass terms:

$$
\begin{aligned}
\mathcal{L}_{\text {Yuk }}= & -\frac{1}{2} m_{03} \nu_{03} \nu_{03}-\frac{1}{2} m_{04} \nu_{04} \nu_{04} \\
& -\frac{1}{\sqrt{2}} d_{0}\left(H_{0}+i A_{0}\right) \nu_{02}\left(-i s_{034} \nu_{03}+c_{034} \nu_{04}\right) \\
& -\frac{1}{\sqrt{2}}\left[y_{0}\left(h_{0}+i \chi_{\mathrm{Z} 0}\right)+d_{0}^{\prime}\left(H_{0}+i A_{0}\right)\right] \\
& \times\left[c_{034} s_{034} \nu_{03} \nu_{03}+i\left(c_{034}^{2}-s_{34}^{2}\right) \nu_{03} \nu_{04}\right. \\
& \left.+c_{034} s_{034} \nu_{04} \nu_{04}\right]+ \text { H.c. }
\end{aligned}
$$

We straightforwardly apply the multiplicative renormalization constants, Eq. (4), for all the parameters and fields. The tree level renormalized effective action is then written in the same way as the bare Lagrangian, except that the parameters and fields are the renormalized ones:

$$
\begin{aligned}
\hat{\Gamma}_{\text {Yuk }}^{[0]}= & -\frac{1}{2} m_{3} \nu_{3} \nu_{3}-\frac{1}{2} m_{4} \nu_{4} \nu_{4} \\
& -\frac{1}{\sqrt{2}} d(H+i A) \nu_{2}\left(-i s_{34} \nu_{3}+c_{34} \nu_{4}\right) \\
& -\frac{1}{\sqrt{2}}\left[y\left(h+i \chi_{Z}\right)+d^{\prime}(H+i A)\right] \\
& \times\left[c_{34} s_{34} \nu_{3} \nu_{3}+i\left(c_{34}^{2}-s_{34}^{2}\right) \nu_{3} \nu_{4}+c_{34} s_{34} \nu_{4} \nu_{4}\right] \\
& + \text { H.c... }
\end{aligned}
$$

where:

$$
\begin{array}{rlrl}
M & =m_{4}-m_{3}, & y^{2} v^{2}=2 m_{3} m_{4} . \\
s_{34}^{2} & =\frac{m_{3}}{m_{4}+m_{3}}, \quad c_{34}^{2}=\frac{m_{4}}{m_{4}+m_{3}} .
\end{array}
$$

Having Eqs. (38) and (39) for the bare theory and Eqs. (42) and (43) for the renormalized one gives us the relations between the renormalization constants:

$$
\begin{gathered}
\delta_{m 3}+\delta_{m 4}=2\left(\delta_{v}+\delta_{y}\right), \\
m_{4} \delta_{m 4}-m_{3} \delta_{m 3}=\left(m_{4}-m_{3}\right) \delta_{M} .
\end{gathered}
$$

The mass renormalization constants are fixed by the CMS condition [24]:

$\delta_{m i}=\left.\frac{1}{2}\left(\Sigma_{\nu_{i} \nu_{i}}+\Sigma_{\bar{\nu}_{i} \bar{\nu}_{i}}+\Sigma_{\nu_{i} \bar{\nu}_{i}}+\Sigma_{\bar{\nu}_{i} \nu_{i}}\right)\right|_{p^{2}=m_{i}^{2}}, \quad m_{i} \neq 0$,

which is nothing more than the usual expression for the OS renormalized mass counterterm (as in [36]) extended to the complex domain and written in Weyl spinor formalism. The CMS condition gives us the renormalized mass parameters gauge independent, however from Eq. (44) we see that the mass counterterm has the $\delta_{v}$ contribution, which is gauge dependent. Hence in this way the bare masses become gauge dependent as well.

Recalling the discussion at the end of Sec. III: to define the gauge invariant mass counterterm we need to identify the bare mass with the proper VEV [31]. Thus the bare relation Eq. (38) is modified to:

$$
M_{0}=m_{04}^{\prime}-m_{03}^{\prime}, \quad y_{0}^{2} v^{2}=2 m_{04}^{\prime} m_{03}^{\prime},
$$

so that there is no $\delta_{v}$ in the definition of $\delta_{m}^{\prime}$ s. From $v_{0}=$ $v\left(1+\delta_{v}\right)$ and comparing Eq. (38) with Eq. (47), we get the relationship between primed (FJ scheme) and unprimed (usual tadpole scheme) mass parameters:

$$
m_{0 i}=m_{0 i}^{\prime}+\Delta_{0}, \quad \Delta_{0}=2 \frac{m_{04}^{\prime} m_{03}^{\prime} \delta_{v}}{m_{04}^{\prime}+m_{03}^{\prime}}, \quad i=3,4 .
$$

As the seesaw mixing parameters depend on the masses, they are shifted as well:

$$
\begin{aligned}
& s_{034}^{2} \rightarrow s_{034}^{2}+2 \delta_{v} c_{034}^{2} s_{034}^{2}\left(c_{034}^{2}-s_{034}^{2}\right), \\
& c_{034}^{2} \rightarrow c_{034}^{2}-2 \delta_{v} c_{034}^{2} s_{034}^{2}\left(c_{034}^{2}-s_{034}^{2}\right) .
\end{aligned}
$$

However, these shifts of the mixing parameters become relevant only at higher loops than one, so we can drop them from our one loop expressions. At one loop level, everything is the same as in Eq. (40), except that the bare mass term Lagrangian for neutrinos becomes: 
$\mathcal{L}_{\text {mass }}=-\frac{1}{2}\left(m_{03}^{\prime}+\Delta_{0}\right) \nu_{03} \nu_{03}-\frac{1}{2}\left(m_{04}^{\prime}+\Delta_{0}\right) \nu_{04} \nu_{04}$.

Starting from this bare Lagrangian, Eq. (46) is modified to:

$$
\begin{aligned}
\delta_{m i}^{\prime}= & \left.\frac{1}{2}\left(\Sigma_{\nu_{i} \nu_{i}}+\Sigma_{\bar{\nu}_{i} \bar{\nu}_{i}}+\Sigma_{\nu_{i} \bar{\nu}_{i}}+\Sigma_{\bar{\nu}_{i} \nu_{i}}\right)\right|_{p^{2}=m_{i}^{2}}-\frac{\Delta}{m_{i}}, \\
& \text { for } m_{i} \neq 0,
\end{aligned}
$$

where:

$$
\Delta=2 \frac{m_{3} m_{4} \delta_{v}}{m_{4}+m_{3}}
$$

is defined with the renormalized masses $m_{3}$ and $m_{4}$. We see that $\Delta$ is the same for $\nu_{3}$ and $\nu_{4}$. To check if $\Delta$ cancels the gauge invariance, we analytically calculate the gauge dependent parts of Eqs. (46) and (52) for $\nu_{3}$ and $\nu_{4}$. Note that in both tadpole schemes the renormalized masses are the same CMS masses, while the bare masses $m_{0 i}$ differ from $m_{0 i}^{\prime}$ by $\Delta_{0}$ as in Eq. (48).

\section{ARRIVING AT THE EXPRESSIONS FOR RENORMALIZATION CONSTANTS}

We use FEynARTs [22] and FormCALC [23] to arrive at one loop expressions for self energies and tadpoles. For making the FEYNARTS model file we found the SARAH [20] package to be useful, which allows to quickly generate a model file from an input of the Lagrangian in terms of Weyl spinors and scalars in the user specified gauge group representations. It also has some built in functions to check the consistency of the model. We choose the Higgs basis by simply putting the VEV of the second Higgs doublet to zero in the input file. We leave all the other parameters arbitrary for generating the FEYNARTS model file and make replacement rules for the FEYNARTS model file parameters to implement our parametrization afterwards. As we work at the one loop level, tree level relations to simplify one loop diagrams can be used. As discussed in Sec. II, the CMS keeps the algebraic structure of the bare theory. This means that for the algebraic simplifications, all the properties and the relations of bare parameters can be used for the renormalized parameters in the CMS as well. Hence we can implement these properties and relations into the assumptions of the Mathematica file in which we do these simplifications. Then the results can be consistently continued to the complex domain afterwards. In the following subsection we show how we implemented the parametrizations into the FEYNARTS model file and the assumptions for the bare parameters that carry over to the algebraic one loop simplifications. Then we present the results that we got for the gauge dependent terms in mass and tadpole renormalization.

\section{A. Getting FeynArts model file}

(1) We generate a FEYNARTS model file using SARAH: (i) We take a SARAH model file for a $2 \mathrm{HDM}$, and define 1 additional gauge singlet like this:

\begin{tabular}{|l|}
\hline Fermionfields $[[6]]=$ \\
$\{\mathrm{n}, 1, \operatorname{conj}[\mathrm{nR}], 0,1,1\}$
\end{tabular}

where the last three entries are the charges under the gauge groups (singlets under all of them), the second is the number of families, the first and the third is the name of the field and its component, respectively (see [20]).

(ii) We modify the Yukawa Lagrangian of that model file to include the general Yukawa couplings of neutrinos with the first and the second Higgs doublet as in Eq. (32) in a direct analog to the quark sector and add the Majorana mass term for the sterile neutrino:

LagYukawan $=-(-$ Yn1 H1.n.1
-Yn2 H2.n.1 + 1/2 Mn.n $)$

(iii) In the definitions for the "EWSB" phase, we set the VEV of the second Higgs doublet to zero to implement the Higgs basis as in Eq. (16):

\begin{tabular}{|l|}
\hline DEFINITION[EWSB] [VEVs] $=$ \\
$\{\{$ H10, $\{\mathrm{v}, 1 /$ Sqrt $[2]\}$, \\
$\{$ sigma1, $\backslash[$ ImaginaryI]/Sqrt $[2]\}$, \\
$\{$ phi1,1/Sqrt $[2]\}\}$, \\
$\{$ H2 0, $\{0,1 /$ Sqrt $[2]\}$, \\
$\{$ sigma2, $\backslash$ ImaginaryI]/Sqrt $[2]\}$, \\
$\{$ phi2,1/Sqrt $[2]\}\}\} ;$
\end{tabular}

(iv) We leave the definition of mixing between Higgses $h$ and $H$ as in the 2HDM model, but omit mixings between the pseudoscalars and the charged scalars as they do not appear in the Higgs basis with $C P$ conserved potential.

(v) We define an additional mixing matrix for neutrinos in the DEFINITION [EWSB] [MatterSector], combining the flavor basis SM neutrinos vL with the sterile neutrino $\operatorname{conj}(\mathrm{nR})$ as:

$\{\{\mathrm{vL}, \mathrm{conj}[\mathrm{nR}]\},\{\mathrm{VL}, \mathrm{Un}\}\}$

where the VL is the combined four-vector of the neutrino mass eigenstates and Un is the mixing matrix $U^{*}$ from Eq. (37).

(vi) We generate the FEYNARTS model file by the SARAH command MakeFeynArts [] . 
(2) We make modifications to the FEYNARTs model file:

(i) To achieve the parametrization of Eq. (34) we make the replacements in the model file for the neutrino-neutrino-Higgs vertices:

$$
\begin{gathered}
\sum_{j=1}^{3} U_{i j} Y_{j}^{1} \rightarrow\left(0,0,-i c_{34} y, s_{34} y\right)_{j}, \\
\sum_{j=1}^{3} U_{i j} Y_{j}^{2} \rightarrow\left(0, d,-i c_{34} d^{\prime}, s_{34} d^{\prime}\right)_{j} .
\end{gathered}
$$

We do not replace the neutrino-electronscalar vertices, hence they depend on $Y^{1}$ and $Y^{2}$ instead of the $y, d$ and $d^{\prime}$ parameters in the model file. We leave them general, because it is easier to make algebraic simplifications of amplitudes in the general couplings for these vertices. After the expressions are simple enough, we invert Eqs. (53) and (54) to express $Y^{1}$ and $Y^{2}$ in terms of $U, y, d$ and $d^{\prime}$ in the Mathematica notebook file.

After setting up the FEYNARTS model file, we generate 1 loop diagrams for the wanted correlation functions. The parametrizations and relations of Secs. III and IV are imposed as replacement rules during the algebraic simplifications of the expressions. The summary of the parameters and their relations is given in the Appendix A.

\section{B. Mass renormalization}

We construct the mass renormalization constants as in Eq. (46) to isolate the gauge dependent part so that we can later check if the definition in Eq. (51) really cancels it. The FormCalc output is easy to use in Weyl spinor notation as the spinor products in the result of the amplitude appear in "WeylChains". By collecting terms near those "WeylChains" we can take separately all four components presented in Eq. (3). The structure of the correction to a propagator is:

$$
\left\langle\nu_{i} \nu_{i}\right\rangle \Gamma_{\nu_{i} \nu_{i}}+\left\langle\bar{\nu}_{i} \bar{\nu}_{i}\right\rangle \Gamma_{\bar{\nu}_{i} \bar{\nu}_{i}}+\left\langle\nu_{i} p \sigma \bar{\nu}_{i}\right\rangle \Sigma_{\nu_{i} \bar{\nu}_{i}}+\left\langle\bar{\nu}_{i} p \bar{\sigma} \nu_{i}\right\rangle \Sigma_{\bar{\nu}_{i} \nu_{i}} .
$$

For Majorana particles only two of the scalar self energies are independent, since $\Sigma_{\nu \bar{\nu}}$ is the same as $\Sigma_{\bar{\nu} \nu}$ and $\Gamma_{\nu \nu}$ is related to $\Gamma_{\bar{\nu} \bar{\nu}}$. At one loop, this relation is just the Hermitian conjugation of couplings that enter the loop functions.

To make algebra simplifications easier and faster we separate different one loop contributions to self energies according to the particles that appear in the loop. Those contributions are from the neutral Higgs scalars, the charged scalar Higgs, the neutral Goldstone boson, the charged Goldstone boson, the $\mathrm{W}$ boson and the $\mathrm{Z}$ boson. We label them as $\Sigma^{H 0}, \Sigma^{H+}, \Sigma^{\chi 0}, \Sigma^{\chi+}, \Sigma^{W}$ and $\Sigma^{Z}$, respectively. Note that the $\Sigma$ s are the dimensionless one loop self energy functions defined in Eq. (14). Analogously, we write the dimensionful self energies as $\Gamma_{\phi_{1} \phi_{2}}^{H 0}, \Gamma_{\phi_{1} \phi_{2}}^{H+}$, etc... Naturally, $\Gamma_{\nu_{i} \nu_{j}}^{H 0}$ and $\Gamma_{\nu_{i} \nu_{j}}^{H+}$ do not depend on any gauge parameter. As the first results of the calculations give us:

$$
\Gamma_{\nu_{1} \nu_{1}}^{[1]}=0 \quad \text { and } \quad \Gamma_{\nu_{2} \nu_{2}}^{[1]}=\Gamma_{\nu_{2} \nu_{2}}^{H 0} .
$$

Note that $\nu_{2}$ and $\nu_{1}$ do not have mass renormalization constants coming from Eq. (4), since they do not have bare mass parameters. The nonvanishing contribution for the mass of $\nu_{2}$ is gauge independent and finite. This is a good first crosscheck to see that the implementation of the model gives us expected results.

We are interested in the gauge dependent part of $\delta_{m 3}$ and $\delta_{m 4}$, so we are interested only in $\Sigma^{\chi^{0}}, \Sigma^{\chi+}, \Sigma^{W}$, and $\Sigma^{Z}$. $\xi_{W}$ will appear only in $\Sigma^{\chi+}$ and $\Sigma^{W}$ and $\xi_{Z}$ only in $\Sigma^{\chi^{0}}$ and $\Sigma^{Z}$. As one can check, the charged loop for masslike terms vanishes:

$$
\Gamma_{\nu_{3} \nu_{3}}^{W}=\Gamma_{\nu_{3}^{\dagger} \nu_{3}^{\dagger}}^{W}=0 .
$$

Hence the potentially $\xi_{W}$ dependent contribution for $m_{3} \delta_{m 3}$ is

$$
\frac{1}{2}\left(\Gamma_{\nu_{3} \nu_{3}}^{\chi+}+\Gamma_{\nu_{3}^{\dagger} \nu_{3}^{\dagger}}^{\chi+}\right)+m_{3} \Sigma_{\nu_{3} \nu_{3}^{\dagger}}^{W}+m_{3} \Sigma_{\nu_{3} \nu_{3}^{+}}^{\chi+}
$$

After some effort (see the Appendix B), we arrive at the $\xi_{W}$ dependent part of the mass counterterm [recall Eq. (5)]:

$$
m_{3} \delta_{m_{3} \xi_{W}}=\frac{m_{3} m_{4}}{\left(m_{3}+m_{4}\right)} \frac{g_{e}^{2}}{16 \pi^{2} m_{Z}^{2} s_{2 W}^{2}} 2 A_{0}\left(m_{W}^{2} \xi_{W}\right),
$$

where $s_{2 W} \equiv 2 s_{W} c_{W}$ is the sine of a double Weinberg angle Eq. (A5). For calculating $\delta_{m_{3} \xi_{z}}$ one should note that $\Gamma_{\nu_{3} \nu_{3}}^{Z} \neq 0$. Apart from that, everything is analogous to the $\xi_{W}$ case. At the end the full gauge dependence of the neutrino mass counterterms is

$$
\begin{aligned}
m_{3} \delta_{m_{3} \xi} & =m_{4} \delta_{m_{4} \xi} \\
& =\frac{m_{3} m_{4}}{\left(m_{3}+m_{4}\right)} \frac{g_{e}^{2}}{16 \pi^{2} m_{Z}^{2} s_{2 W}^{2}}\left[A_{0}\left(m_{Z}^{2} \xi_{Z}\right)+2 A_{0}\left(m_{W}^{2} \xi_{W}\right)\right] .
\end{aligned}
$$

\section{VEV renormalization}

When separating the gauge parameter dependent part of $T_{h}^{[1]}$ we first observe that tadpoles with physical Higgs bosons and fermions in the loop do not have any gauge dependence. The gauge dependent part of loops with gauge bosons and ghosts exactly cancel when these contributions are summed up. Hence the only gauge dependent terms in the tadpole contributions are the tadpoles with Goldstone bosons in the loops, which are

$$
T_{h \xi}^{[1]}=\frac{\lambda_{1} v}{32 \pi^{2}}\left[A_{0}\left(m_{Z}^{2} \xi_{Z}\right)+2 A_{0}\left(m_{W}^{2} \xi_{W}\right)\right] .
$$


This is exactly the same term that we would get for the Higgs tadpole in the SM. This again shows the convenience of the Higgs basis in the tadpole equations. From Eqs. (30) and (52) we have:

$\Delta_{\xi}=\frac{m_{3} m_{4}}{\left(m_{3}+m_{4}\right)} \frac{1}{16 \pi^{2} v^{2}}\left[A_{0}\left(m_{Z}^{2} \xi_{Z}\right)+2 A_{0}\left(m_{W}^{2} \xi_{W}\right)\right]$,

which, inserting the SM relations of Eq. (A5) gives exactly the same result as Eq. (60).

\section{DISCUSSION AND CONCLUSIONS}

We analytically checked in the CMS or the OS scheme that the gauge dependent term of the mass counterterms for the neutrinos of the GN model comes only from the tadpole contributions, Eq. (60), as suggested in [18]. Using multiplicative renormalization constants and the relations between them, shown in Eqs. (44) and (45), we present how the gauge dependence of neutrino mass counterterms can be seen as a contribution coming from $\delta_{v}$, the renormalization constant of the VEV in the usual tadpole renormalization (e.g., [36]). We also get that this tadpole contribution is the same for both neutrino counterterms:

$$
m_{3} \delta_{m_{3} \xi}=m_{4} \delta_{m_{4} \xi}=\Delta_{\xi} .
$$

This is one of the features of the GN model: the single sterile neutrino leads to the single value of the Yukawa coupling $y$ to the first Higgs doublet in the Higgs basis. This single value is coupled to the VEV, hence only the single value $\Delta$, related to the VEV shift $\delta_{v}$, is possible for the neutrino mass counterterms in this setup.

The alternative tadpole scheme, or the FJ scheme [31], consistently omits this gauge dependence from the mass renormalization constants by identifying the bare masses with the proper VEV. Following this scheme, we modify the definition of the mass counterterms to include this tadpole contribution in Eq. (51). This definition now exactly cancels the gauge dependent contribution as can be seen from Eq. (63). The factor $\Delta$ gives the same contribution for the mass counterterms as if we would add the contribution of diagrams with tadpoles connected to the propagators as in [18]. The fact that the procedures of [31] works for the seesaw neutrinos just in the same way as with the Dirac particles is explained by the fact that only the Dirac mass $\left[\sim m_{3} m_{4}\right.$ from Eq. (38)] is directly related to the VEV. The other crosscheck is that the result of Eq. (63), using Eq. (45), gives

$$
\delta_{M \xi}=0,
$$

or in other words, the Majorana mass term $M$, does not acquire gauge dependence in any of these schemes. This again confirms the statement that the Majorana mass term of the sterile part of the neutrino does not affect the application of the FJ scheme for mass counterterms for the neutrinos. Hence using the $\mathrm{FJ}$ scheme is straightforwardly applicable in the GN model.

\section{ACKNOWLEDGMENTS}

The authors thank the Lithuanian Academy of Sciences for the support (the Project No. DaFi2017).

\section{APPENDIX A: PARAMETRIZATIONS, ASSUMPTIONS, AND RELATIONS}

Here we collect all parameters and relations used in our 1 loop calculations. The assumption that some bare parameter $p_{0}$ is real, is reflected in the renormalized theory in the sense of Eq. (13). In the FormCALC output for one loop corrections for masses, we implement this assumption by the replacement rule $p^{\dagger} \rightarrow p$, for $p_{0} \in \mathbb{R}$.

\section{Scalar sector and the SM relations}

The assumptions of $C P$ conservation of the Higgs potential give:

$$
m_{0 i j}^{2}, \lambda_{0 k} \in \mathbb{R} ; \quad i, j=1,2, \quad k=1, \ldots, 7 .
$$

The minimum conditions are

$$
m_{11}^{2}=-\frac{1}{2} \lambda_{1} v^{2} \quad \text { and } \quad m_{12}^{2}=\frac{1}{2} \lambda_{6} v^{2} .
$$

The Higgs basis is given by:

$H_{1}=\left(\begin{array}{c}\chi_{0 W}^{+} \\ \frac{1}{\sqrt{2}}\left(v+h+i \chi_{Z}\right)\end{array}\right), \quad H_{2}=\left(\begin{array}{c}H_{0}^{+} \\ \frac{1}{\sqrt{2}}(H+i A)\end{array}\right)$.

The mixing matrix for scalars is only between $h$ and $H$ :

$$
\begin{aligned}
& O^{\phi}=\left(\begin{array}{cc}
c_{\alpha} & s_{\alpha} \\
-s_{\alpha} & c_{\alpha}
\end{array}\right), \quad \phi_{i}^{\text {mass }}=O_{i j}^{\phi} \phi_{j}^{\text {Higgs }}, \\
& \phi_{i}^{\text {Higgs }}=(h, H)_{i},
\end{aligned}
$$

where $s_{\alpha}, c_{\alpha}$ are sine and cosine functions of the mixing angle $\alpha$.

The relations of the electroweak sector are

$s_{2 W} \equiv 2 s_{W} c_{W}, \quad m_{Z}=\frac{g_{e} v}{s_{2 W}}, \quad m_{W}=m_{Z} c_{W}$,

where $s_{W}$ and $c_{W}$ are sine and cosine functions of Weinberg angle.

\section{Yukawa sector}

As the first thing after generating the FEYNARTs model file we make the replacements Eqs. (53) and (54):

$$
\begin{aligned}
& \sum_{j=1}^{3} U_{i j} Y_{j}^{1} \rightarrow\left(0,0,-i c_{34} y, s_{34} y\right)_{j}, \\
& \sum_{j=1}^{3} U_{i j} Y_{j}^{2} \rightarrow\left(0, d,-i c_{34} d^{\prime}, s_{34} d^{\prime}\right)_{j} .
\end{aligned}
$$


The parametrization of Yukawa couplings are summarized as:

$$
\begin{array}{lll}
V_{1 j} Y_{j}^{1}=0, & V_{2 j} Y_{j}^{1}=0, & V_{3 j} Y_{j}^{1}=y_{0}, \\
V_{1 j} Y_{j}^{2}=0, & V_{2 j} Y_{j}^{2}=d_{0}, & V_{2 j} Y_{j}^{2}=d_{0}^{\prime}, \\
d_{0}, y_{0} \in \mathbb{R}, & d_{0}^{\prime} \in \mathbb{C}, &
\end{array}
$$

where the neutrino mixing matrix is

$$
U=U^{34} V=U^{34} U^{\beta} O^{12} U^{\alpha} O^{13} O^{23}
$$

with the relations

$$
\nu_{0 i}^{F}=\left(n_{0 e}, n_{0 \mu}, n_{0 \tau}, N_{0}\right)_{i}, \quad \nu_{0 i}^{\operatorname{mass}}=U_{i j}^{*} \nu_{0 j}^{F} .
$$

The parametrization of the mixing matrix can be written as:

$$
\begin{aligned}
s_{0 i j}^{2}+c_{0 i j}^{2} & =1, \quad s_{0 i j}, c_{0 i j}, \sigma_{0}, \rho_{0} \in \mathbb{R} ; \\
O_{i j}^{A B} & =1_{i j} \text { for } i, j \neq A, B ; \\
O_{A B}^{A B} & =-O_{B A}^{A B}=s_{0 A B} ; \quad O_{A A}^{A B}=O_{B B}^{A B}=c_{0 A B} ; \\
U_{i j}^{\sigma} & =e^{i \sigma_{0}} \text { for } i=j=1 ; \quad U_{i j}^{\sigma}=1_{i j} \text { for } i, j \neq 1 ; \\
U_{i j}^{\rho} & =e^{i \rho_{0}} \text { for } i=j=2 ; \quad U_{i j}^{\rho}=1_{i j} \text { for } i, j \neq 2 ; \\
U_{34}^{34} & =i \cdot U_{43}^{34}=i \cdot s_{034} ; \quad U_{33}^{34}=-i \cdot U_{44}^{34}=-i \cdot c_{034} ; \\
U_{i j}^{34} & =1_{i j} \text { for } i, j \neq 3,4 .
\end{aligned}
$$

The seesaw mechanism is realized with:

$$
\begin{gathered}
M_{0}=m_{04}-m_{03}, \quad y_{0}^{2} v_{0}^{2}=2 m_{03} m_{04}, \\
s_{034}^{2}=\frac{m_{03}}{m_{04}+m_{03}} \quad \text { and } \quad c_{034}^{2}=\frac{m_{04}}{m_{04}+m_{03}} .
\end{gathered}
$$

\section{APPENDIX B: ARRIVING AT EQ. (59)}

Here we show some intermediate steps for arriving at the gauge parameter $\xi_{W}$ dependent term for the $\delta_{m 3}$ counterterm shown in Eq. (59). We start from Eq. (58):

$$
\frac{1}{2}\left(\Gamma_{\nu_{3} \nu_{3}}^{\chi+}+\Gamma_{\nu_{3}^{\dagger} \nu_{3}^{\dagger}}^{\chi+}\right)+m_{3} \Sigma_{\nu_{3} \nu_{3}^{\dagger}}^{W}+m_{3} \Sigma_{\nu_{3} \nu_{3}^{\dagger}}^{\chi+}
$$

Let us first look at the loop with the Goldstone boson $\Gamma_{\nu_{3} \nu_{3}}^{\chi+}\left(m_{3}^{2}\right)$. We set up the model file in FEYNARTS following the steps in Sec. VA. After generating diagrams with FeynArts, creating an amplitude with FormCALC, implementing the parametrization that is summarised in Appendix A by the replacement rules, the standard Mathematica "Simplify" command should give:

$$
\begin{aligned}
\Gamma_{\nu_{3} \nu_{3}}^{\chi+}\left(m_{3}^{2}\right)= & \frac{-\sqrt{m_{3} m_{4}}}{4 \sqrt{2} \pi^{2}\left(m_{3}+m_{4}\right) v}\left[-m_{\tau}^{2} Y_{\tau}^{1 *} c_{13} c_{23} B_{0}\left(m_{3}^{2}, m_{W}^{2} \xi_{W}, m_{\tau}^{3}\right)+m_{\mu}^{2} Y_{\mu}^{1 *} c_{13} s_{23} B_{0}\left(m_{3}^{2}, m_{W}^{2} \xi_{W}, m_{\mu}^{3}\right)\right. \\
& \left.+m_{e}^{2} Y_{e}^{1 *} s_{13} B_{0}\left(m_{3}^{2}, m_{W}^{2} \xi_{W}, m_{e}^{3}\right)\right] .
\end{aligned}
$$

Expressing $Y^{1}$ from Eqs. (53) and (54) gives:

$$
\begin{aligned}
\Gamma_{\nu_{3} \nu_{3}}^{\chi+}\left(m_{3}^{2}\right)= & \frac{-y \sqrt{m_{3} m_{4}}}{4 \sqrt{2} \pi^{2}\left(m_{3}+m_{4}\right) v}\left[m_{\tau}^{2} c_{13}^{2} c_{23}^{2} B_{0}\left(m_{3}^{2}, m_{W}^{2} \xi_{W}, m_{\tau}^{3}\right)\right. \\
& \left.+m_{\mu}^{2} c_{13}^{2} s_{23}^{2} B_{0}\left(m_{3}^{2}, m_{W}^{2} \xi_{W}, m_{\mu}^{3}\right)+m_{e}^{2} s_{13}^{2} B_{0}\left(m_{3}^{2}, m_{W}^{2} \xi_{W}, m_{e}^{3}\right)\right] .
\end{aligned}
$$

Now we can express $v$ in terms of Eq. (A5) and $y$ in terms of Eq. (42) to get:

$$
\begin{aligned}
\Gamma_{\nu_{3} \nu_{3}}^{\chi+}\left(m_{3}^{2}\right)= & \frac{-g_{e}^{2} m_{3} m_{4}}{4 \pi^{2}\left(m_{3}+m_{4}\right) m_{Z}^{2} s_{2 W}^{2}}\left[m_{\tau}^{2} c_{13}^{2} c_{23}^{2} B_{0}\left(m_{3}^{2}, m_{W}^{2} \xi_{W}, m_{\tau}^{3}\right)\right. \\
& \left.+m_{\mu}^{2} c_{13}^{2} s_{23}^{2} B_{0}\left(m_{3}^{2}, m_{W}^{2} \xi_{W}, m_{\mu}^{3}\right)+m_{e}^{2} s_{13}^{2} B_{0}\left(m_{3}^{2}, m_{W}^{2} \xi_{W}, m_{e}^{3}\right)\right] .
\end{aligned}
$$

The result for $\Gamma_{\nu_{3}^{\dagger} \nu_{3}^{\dagger}}^{\chi+}$ is the same, as it should be, since $\nu_{3}$ is a Majorana fermion and the couplings can be taken real for the one loop correction, hence we can write:

$$
\begin{aligned}
\frac{1}{2}\left(\Gamma_{\nu_{3} \nu_{3}}^{\chi+}+\Gamma_{\nu_{3}^{\dagger} \nu_{3}^{\dagger}}^{\chi+}\right)= & \frac{-g_{e}^{2} m_{3} m_{4}}{4 \pi^{2}\left(m_{3}+m_{4}\right) m_{Z}^{2} s_{2 W}^{2}} \times\left[m_{\tau}^{2} c_{13}^{2} c_{23}^{2} B_{0}\left(m_{3}^{2}, m_{W}^{2} \xi_{W}, m_{\tau}^{3}\right)\right. \\
& \left.+m_{\mu}^{2} c_{13}^{2} s_{23}^{2} B_{0}\left(m_{3}^{2}, m_{W}^{2} \xi_{W}, m_{\mu}^{3}\right)+m_{e}^{2} s_{13}^{2} B_{0}\left(m_{3}^{2}, m_{W}^{2} \xi_{W}, m_{e}^{3}\right)\right] .
\end{aligned}
$$


We follow exactly the same steps for $\sum_{\nu_{3} \nu_{3}^{\dagger}}^{\chi+}$ to get:

$$
\begin{aligned}
\frac{g_{e}^{2} m_{4}}{8 \pi^{2}\left(m_{3}+m_{4}\right) m_{Z}^{2} s_{2 W}^{2}}[ & m_{3}^{2} c_{13}^{2} c_{23}^{2} B_{0}\left(m_{3}^{2}, m_{W}^{2} \xi_{W}, m_{\tau}^{2}\right)+m_{3}^{2} c_{13}^{2} s_{23}^{2} B_{0}\left(m_{3}^{2}, m_{W}^{2} \xi_{W}, m_{\mu}^{2}\right)+m_{3}^{2} s_{13}^{2} B_{0}\left(m_{3}^{2}, m_{W}^{2} \xi_{W}, m_{e}^{2}\right) \\
& +m_{\tau}^{2} c_{13}^{2} c_{23}^{2} B_{0}\left(m_{3}^{2}, m_{W}^{2} \xi_{W}, m_{\tau}^{2}\right)+m_{\mu}^{2} c_{13}^{2} s_{23}^{2} B_{0}\left(m_{3}^{2}, m_{W}^{2} \xi_{W}, m_{\mu}^{2}\right)+m_{e}^{2} s_{13}^{2} B_{0}\left(m_{3}^{2}, m_{W}^{2} \xi_{W}, m_{e}^{2}\right) \\
& +m_{3}^{2} c_{13}^{2} c_{23}^{2} B_{1}\left(m_{3}^{2}, m_{W}^{2} \xi_{W}, m_{\tau}^{2}\right)+m_{3}^{2} c_{13}^{2} s_{23}^{2} B_{1}\left(m_{3}^{2}, m_{W}^{2} \xi_{W}, m_{\mu}^{2}\right)+m_{3}^{2} s_{13}^{2} B_{1}\left(m_{3}^{2}, m_{W}^{2} \xi_{W}, m_{e}^{2}\right) \\
& \left.+m_{\tau}^{2} c_{13}^{2} c_{23}^{2} B_{1}\left(m_{3}^{2}, m_{W}^{2} \xi_{W}, m_{\tau}^{2}\right)+m_{\mu}^{2} c_{13}^{2} s_{23}^{2} B_{1}\left(m_{3}^{2}, m_{W}^{2} \xi_{W}, m_{\mu}^{2}\right)+m_{e}^{2} s_{13}^{2} B_{1}\left(m_{3}^{2}, m_{W}^{2} \xi_{W}, m_{e}^{2}\right)\right] .
\end{aligned}
$$

The loop with the $W$ boson $\Sigma_{\nu_{3} \nu_{3}^{\ddagger}}^{W}$ will have gauge invariant contributions from the transverse polarization of the $\mathrm{W}$ boson. These can be dropped out from the expression by formally differentiating and integrating with respect to $\xi_{W}$ in Mathematica. Then every step for simplifying the expression is the same as before with the result:

$$
\begin{aligned}
\frac{g_{e}^{2} m_{4}}{8 \pi^{2}\left(m_{3}+m_{4}\right) m_{Z}^{2} s_{2 W}^{2}}[ & m_{3}^{2} c_{13}^{2} c_{23}^{2} B_{0}\left(m_{3}^{2}, m_{W}^{2} \xi_{W}, m_{\tau}^{2}\right)-m_{3}^{2} c_{13}^{2} s_{23}^{2} B_{0}\left(m_{3}^{2}, m_{W}^{2} \xi_{W}, m_{\mu}^{2}\right)-m_{3}^{2} s_{13}^{2} B_{0}\left(m_{3}^{2}, m_{W}^{2} \xi_{W}, m_{e}^{2}\right) \\
& +m_{\tau}^{2} c_{13}^{2} c_{23}^{2} B_{0}\left(m_{3}^{2}, m_{W}^{2} \xi_{W}, m_{\tau}^{2}\right)+m_{\mu}^{2} c_{13}^{2} s_{23}^{2} B_{0}\left(m_{3}^{2}, m_{W}^{2} \xi_{W}, m_{\mu}^{2}\right)+m_{e}^{2} s_{13}^{2} B_{0}\left(m_{3}^{2}, m_{W}^{2} \xi_{W}, m_{e}^{2}\right) \\
& -m_{3}^{2} c_{13}^{2} c_{23}^{2} B_{1}\left(m_{3}^{2}, m_{W}^{2} \xi_{W}, m_{\tau}^{2}\right)-m_{3}^{2} c_{13}^{2} s_{23}^{2} B_{1}\left(m_{3}^{2}, m_{W}^{2} \xi_{W}, m_{\mu}^{2}\right)-m_{3}^{2} s_{13}^{2} B_{1}\left(m_{3}^{2}, m_{W}^{2} \xi_{W}, m_{e}^{2}\right) \\
& -m_{\tau}^{2} c_{13}^{2} c_{23}^{2} B_{1}\left(m_{3}^{2}, m_{W}^{2} \xi_{W}, m_{\tau}^{2}\right)-m_{\mu}^{2} c_{13}^{2} s_{23}^{2} B_{1}\left(m_{3}^{2}, m_{W}^{2} \xi_{W}, m_{\mu}^{2}\right)-m_{e}^{2} s_{13}^{2} B_{1}\left(m_{3}^{2}, m_{W}^{2} \xi_{W}, m_{e}^{2}\right) \\
& \left.+c_{13}^{2} c_{23}^{2} A_{0}\left(m_{W}^{2} \xi_{W}\right)+c_{13}^{2} s_{23}^{2} A_{0}\left(m_{W}^{2} \xi_{W}\right)+s_{13}^{2} A_{0}\left(m_{W}^{2} \xi_{W}\right)\right] .
\end{aligned}
$$

Comparing Eq. (B7) with Eq. (B6) we notice that the first, third and fourth lines of both expressions cancel and the second line of both equations is the same. Trigonometric functions near the $A_{0}$ integrals in Eq. (B7) sum to one. The sum of Eqs. (B7) and (B6) multiplied by $m_{3}$ then gives

$$
\begin{gathered}
\frac{g_{e}^{2} m_{4} m_{3}}{4 \pi^{2}\left(m_{3}+m_{4}\right) m_{Z}^{2} s_{2 W}^{2}}\left[m_{\tau}^{2} c_{13}^{2} c_{23}^{2} B_{0}\left(m_{3}^{2}, m_{W}^{2} \xi_{W}, m_{\tau}^{2}\right)+m_{\mu}^{2} c_{13}^{2} s_{23}^{2} B_{0}\left(m_{3}^{2}, m_{W}^{2} \xi_{W}, m_{\mu}^{2}\right)+m_{e}^{2} s_{13}^{2} B_{0}\left(m_{3}^{2}, m_{W}^{2} \xi_{W}, m_{e}^{2}\right)\right] \\
+\frac{g_{e}^{2} m_{4} m_{3}}{8 \pi^{2}\left(m_{3}+m_{4}\right) m_{Z}^{2} s_{2 W}^{2}} A_{0}\left(m_{W}^{2} \xi_{W}\right) .
\end{gathered}
$$

The second line cancels with the contribution of the Goldstone loop from Eq. (B5) giving exactly Eq. (59).

[1] K. M. Heeger, Evidence for neutrino mass: A decade of discovery, in Seesaw 25: Proceedings of the International Conference on the Seesaw Mechanism (World Scientific, Singapore, 2004), pp. 65-80, DOI 10.1142/97898127022 10_0005.

[2] E. Molinaro, Type I seesaw mechanism, lepton flavour violation and Higgs decays, J. Phys. Conf. Ser. 447, 012052 (2013).

[3] J. Orloff, S. Lavignac, and M. Cribier, eds., Seesaw 25: Proceedings of the International Conference on the Seesaw Mechanism (World Scientific, Singapore, 2005), DOI 10.11 42/5717.

[4] W. Grimus and H. Neufeld, Radiative neutrino masses in an SU(2) X U(1) model, Nucl. Phys. B325, 18 (1989).

[5] H. E. Haber and G. L. Kane, The search for supersymmetry: Probing physics beyond the standard model, Phys. Rep. 117, 75 (1985).
[6] J.E. Kim, Light pseudoscalars, particle physics and cosmology, Phys. Rep. 150, 1 (1987).

[7] T. D. Lee, A theory of spontaneous $t$ violation, Phys. Rev. D 8, 1226 (1973).

[8] R. D. Peccei and H. R. Quinn, CP Conservation in the Presence of Pseudoparticles, Phys. Rev. Lett. 38, 1440 (1977).

[9] G. C. Branco, P. M. Ferreira, L. Lavoura, M. N. Rebelo, M. Sher, and J. P. Silva, Theory and phenomenology of two-Higgs-doublet models, Phys. Rep. 516, 1 (2012).

[10] P. Gambino and P. A. Grassi, The Nielsen identities of the SM and the definition of mass, Phys. Rev. D 62, 076002 (2000).

[11] A. Denner, S. Dittmaier, M. Roth, and D. Wackeroth, Predictions for all processes $e^{+} e^{-} \rightarrow$ fermions $+\gamma$, Nucl. Phys. B560, 33 (1999).

[12] A. Denner and S. Dittmaier, The complex-mass scheme for perturbative calculations with unstable particles, Nucl. Phys. B, Proc. Suppl. 160, 22 (2006). 
[13] A. Denner, L. Jenniches, J.-N. Lang, and C. Sturm, Gaugeindependent $\overline{\mathrm{MS}}$ renormalization in the 2HDM, J. High Energy Phys. 09 (2016) 115.

[14] L. Altenkamp, S. Dittmaier, and H. Rzehak, Renormalization schemes for the two-Higgs-doublet model and applications to $\mathrm{h} \rightarrow \mathrm{WW} / \mathrm{ZZ} \rightarrow 4$ fermions, J. High Energy Phys. 09 (2017) 134.

[15] M. E. Machacek and M. T. Vaughn, Two loop renormalization group equations in a general quantum field theory. 1 . Wave function renormalization, Nucl. Phys. B222, 83 (1983).

[16] M. Sperling, D. Stöckinger, and A. Voigt, Renormalization of vacuum expectation values in spontaneously broken gauge theories, J. High Energy Phys. 07 (2013) 132.

[17] M. Sperling, D. Stöckinger, and A. Voigt, Renormalization of vacuum expectation values in spontaneously broken gauge theories: Two-loop results, J. High Energy Phys. 01 (2014) 068.

[18] S. Liebler and W. Porod, Electroweak corrections to neutralino and chargino decays into a W-boson in the $(\mathrm{N})$ MSSM, Nucl. Phys. B849, 213 (2011); Erratum, Nucl. Phys.B856, 125(E) (2012).

[19] J. M. Cornwall, J. Papavassiliou, and D. Binosi, The Pinch Technique and its Applications to Non-Abelian Gauge Theories (Cambridge University Press, Cambridge, England, 2010).

[20] F. Staub, SARAH, arXiv:0806.0538.

[21] F. Staub, SARAH 4: A tool for (not only SUSY) model builders, Comput. Phys. Commun. 185, 1773 (2014).

[22] T. Hahn, Generating Feynman diagrams and amplitudes with FeynArts 3, Comput. Phys. Commun. 140, 418 (2001).

[23] T. Hahn and M. Perez-Victoria, Automated one-loop calculations in four and D-dimensions, Comput. Phys. Commun. 118, 153 (1999).

[24] V. Dūdènas and T. Gajdosik, On the renormalization of neutrinos in the seesaw extension of the two-Higgs doublet model, Acta Phys. Pol. B 48, 2243 (2017).
[25] H. K. Dreiner, H.E. Haber, and S.P. Martin, Twocomponent spinor techniques and Feynman rules for quantum field theory and supersymmetry, Phys. Rep. 494, 1 (2010).

[26] B. A. Kniehl and A. Sirlin, Pole mass, width, and propagators of unstable fermions, Phys. Rev. D 77, 116012 (2008).

[27] B. A. Kniehl, Propagator mixing renormalization for Majorana fermions, Phys. Rev. D 89, 116010 (2014).

[28] D. Espriu, J. Manzano, and P. Talavera, Flavor mixing, gauge invariance and wave function renormalization, Phys. Rev. D 66, 076002 (2002).

[29] S. Davidson and H. E. Haber, Basis-independent methods for the two-Higgs-doublet model, Phys. Rev. D 72, 035004 (2005); Erratum, Phys. Rev. D72, 099902(E) (2005).

[30] H. E. Haber and D. O'Neil, Basis-independent methods for the two-Higgs-doublet model. II. The significance of $\tan \beta$, Phys. Rev. D 74, 015018 (2006); Erratum, Phys. Rev. D74, 059905(E) (2006).

[31] J. Fleischer and F. Jegerlehner, Radiative corrections to Higgs decays in the extended Weinberg-Salam model, Phys. Rev. D 23, 2001 (1981).

[32] S. Actis, A. Ferroglia, M. Passera, and G. Passarino, Two-loop renormalization in the standard model. Part I: Prolegomena, Nucl. Phys. B777, 1 (2007).

[33] M. Krause, On the renormalization of the two-Higgs-doublet model, Master's thesis, KIT, Karlsruhe, TP, 2016. https:// www.itp.kit.edu/_media/publications/masterthesismarcel .pdf.

[34] M. Krause, R. Lorenz, M. Muhlleitner, R. Santos, and H. Ziesche, Gauge-independent renormalization of the 2-Higgs-doublet model, J. High Energy Phys. 09 (2016) 143.

[35] M. Krause, D. Lopez-Val, M. Muhlleitner, and R. Santos, Gauge-independent Renormalization of the N2HDM, J. High Energy Phys. 12 (2017) 077.

[36] A. Denner, Techniques for calculation of electroweak radiative corrections at the one loop level and results for W physics at LEP-200, Fortschr. Phys. 41, 307 (1993). 